\title{
Brightness Temperature Changes From Satellite Infrared Information before Wen'an Earthquake
}

\author{
Yaling Ning ${ }^{1,2, a}$ \\ ${ }^{1}$ Earthquake Administration of Shanxi Province, \\ Tai yuan 030021, China \\ ${ }^{2}$ Continental Rift Valley Dynamics State Observatory of \\ Taiyuan, \\ Tai yuan 030021, China \\ any1000@qq.com \\ Haijie $\mathrm{Lv}^{3, \mathrm{~b}}$ \\ ${ }^{3}$ Zhengzhou Trade and Industy Schools, \\ Zhengzhou 450007, China \\ b70674753@qq.com
}

\author{
Congxin $\mathrm{Wei}^{4,5, \mathrm{c}}$ \\ ${ }^{4}$ Lanzhou Institute of Seismology, \\ China Earthquake Administration, \\ Lanzhou 730000, China \\ ${ }^{5}$ Lanzhou Base of Institute of Earthquake Prediction, \\ China Earthquake Administration, \\ Lanzhou 730000, China \\ cweicx12@163.com \\ Jiashu $\mathrm{Xu}^{6, \mathrm{~d}}$ \\ ${ }^{6}$ College of Geo-Exploration Science and Technology, \\ Jilin University, \\ Changchun 130026, China \\ d12721400@qq.com
}

\begin{abstract}
Using MODIS thermal infrared data of satellite and geostationary satellite thermal infrared brightness temperature data for Wen ' an 5.1 earthquake in Hebei province during 2006, the temporal and spatial characteristics of the earthquake were systematically calculated and analysed. Studies show that the anomaly value does exist in both the heat penetrability index and the anomaly ratio about a month before the earthquake. During the earthquake, the process of the thermal infrared anomaly change had experienced from large-range to concentrated then gradually to dissipation, and the maximum value appeared closely near the epicenter.
\end{abstract}

Keywords-Wen ' an earthquake; satellite thermal infrared; brightness temperature change.

\section{INTRODUCTION}

With the characteristics of abruptness, instantaneity, destructiveness and continuity of earthquake-induced disasters, earthquake is one of serious natural disasters, which brings huge threat to people's lives, and often results in enormously losses of casualties and property to the human society. Hence, countries all over the world are impelled to attach great importance and urgency to seismological study and earthquake prediction. So far, none of any definite seismic precursors had been unexceptionally observed by any seismologist before the great earthquakes occurred. Judging from the prospects of current technology and science, we may use the space satellites to help and catch some valuable information before the great earthquakes occurred. Therefore, using the satellite remote sensing technology may help to overcome the problem of the short-term and impending earthquake prediction.

$\mathrm{Lu}$ et al. studied the possible satellite thermal infrared anomaly before the Zhangbei MS6.2 earthquake. the results present that the thermal infrared anomaly started more than ten days earlier before the earthquake, and the peak value of temperature variation appeared one or two days before the earthquake. They also found that the thermal infrared anomaly had a band distribution, and the temperature increased mainly along faults [1]. Ma et al. also found that the temperature became higher in the eastern Altun fault 20 days earlier before the great 1997 Mani MS7.9 earthquake. The anomaly developed and distributed gradually in belts till the earthquake occurred on 8 December, and then disappeared after the earthquake. However, it was not until 2 days before the earthquake that the temperature increase anomaly appeared in the Margaichaka fault, which induced the earthquake [2].

From the actual application of the satellite remote sensing thermal infrared, the temperature anomaly of different degrees does exist near epicentral area [3-6], which provides practical basis for the application of the satellite remote sensing thermal infrared in earthquake prediction. Therefore, further research in relation of the satellite infrared information and the moderate-strong earthquake can be helpful for getting new understanding and progress, and also has promotive effect on earthquake prediction. Based on the previous studies by others, we studied the thermal anomaly data of the Wen'an MS5.1 earthquake of 2006 .

\section{STUDY AREA AND DATA}

The study area, about $600 \mathrm{~km} * 600 \mathrm{~km}$, is nearly bounded by $36.7^{\circ} \mathrm{N}-46.1^{\circ} \mathrm{N}$ and $111.1^{\circ} \mathrm{E}-118.7^{\circ} \mathrm{E}$ (Fig. 1), which is selected as the analysis range for temporal characteristic method. In this paper, we applied the MODIS thermal infrared data of TERRA satellite, from May 12th, 2004 to December 31st,2006, and selected the data of geostationary satellite forthe spatial characteristics analysis. 


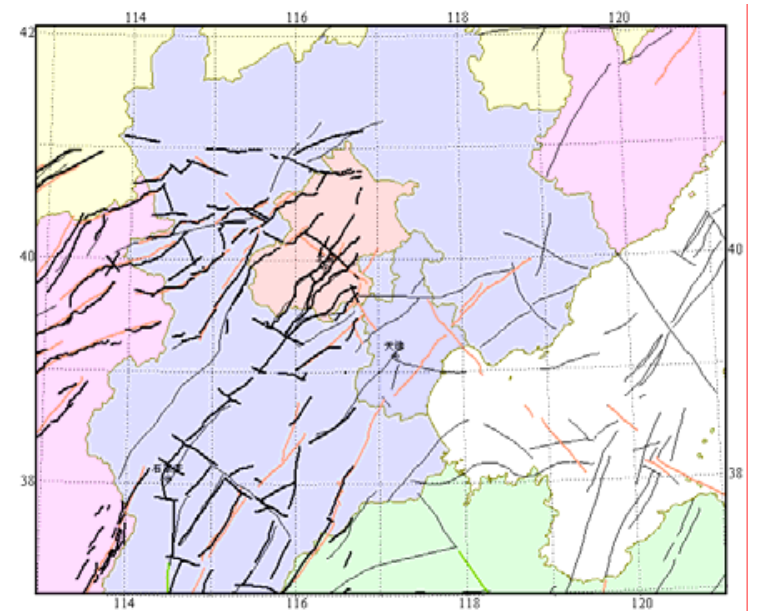

Fig.1. Sketch map of the research area

\section{DAta Processing Methods}

As we know, the satellite remote sensing information is effected by the atmosphere, so does the land surface brightness temperature, which is also under the influence of underground activities. Hence, the heat penetrability index [7] was employed to extract the underground information. The formula of the heat penetrability index (D) is

$$
\mathrm{D}=1-\frac{\left|\sum_{\mathrm{i}=1}^{\mathrm{n}}(\mathrm{u}-\overline{\mathrm{u}}) \sum_{\mathrm{i}=1}^{\mathrm{n}}(\mathrm{v}-\overline{\mathrm{v}})\right|}{\sqrt{\sum_{\mathrm{i}=1}^{\mathrm{n}}(\mathrm{u}-\overline{\mathrm{u}})^{2} \sum_{\mathrm{i}=1}^{\mathrm{n}}\left(\mathrm{v}^{-} \overline{\mathrm{v}}\right)^{2}}},
$$

where $\mathrm{u}$ and $\mathrm{v}$ represent the temperature of two areas, respectively. and $\mathrm{n}$ is the number of samples in the time window. The value of $\mathrm{D}$ is taken as the integer from 0 to 1 .

Furthermore, we made statistical analysis of the brightness temperature increase change of every detection pixel in study area, and then extracted the information of temperature increase anomaly. Based on these, a distribution map and the time-sequence ratio curve of temperature anomaly were exported. For the brightness temperature data of some selected pixels, the mean square deviation $\sigma, \sigma=\sqrt{\frac{\sum\left(\mathrm{T}_{\mathrm{i}}-\overline{\mathrm{T}}\right)^{2}}{\mathrm{n}}},(\mathrm{i}=1,2, \ldots, \mathrm{n})$, was calculated, where $\mathrm{n}$ denotes the length of the sliding time window, $\mathrm{T}$ is the brightness temperature. If $\mathrm{T}$ is greater than the $\mathrm{m}$-fold $\sigma$, we can confirm that it is positive anomaly. Therefore, the ratio of the number of positive anomaly in the total effective number in selected area could be calculated.

\section{ANOMALY ANALYSIS}

Temporal Characteristics of Anomaly

There are mainly three cases for the heat penetrability index and the anomaly ratio:
(1) The heat penetrability index showed abnormal while the anomaly ratio had no anomaly. As Fig. 2 shows, the heat penetrability indices increased rapidly in a couple of days and then went down stably. During this time, there were neither brightness temperature increase anomaly in the study area, nor any earthquake afterwards. In this case, it indicated that there might be some tectonic movement in the area, which somehow didn't cause the earthquake. Further research is still needed to study the relation between the tectonic movement and the earthquake activity in the long future.

(2) High value occurred in the anomaly ratio whereas the heat penetrability index unchanged. As it is shown in Fig. 3 that high value occurred continuously in several time segments, which indicated that the number of the surface brightness temperature abnormal areas increased considerably. Meanwhile, the heat penetrability index had no change, which explains that the anomaly was caused by temperature change instead of tectonic movement.

(3) There were anomalies in both the heat penetrability index and the anomaly ratio as shown in Fig.4. The surface brightness temperature change was under the influence of underground tectonic movement which also caused the surface brightness temperature increasing. In this case, moderate earthquake may happen in a future month in the area or around.

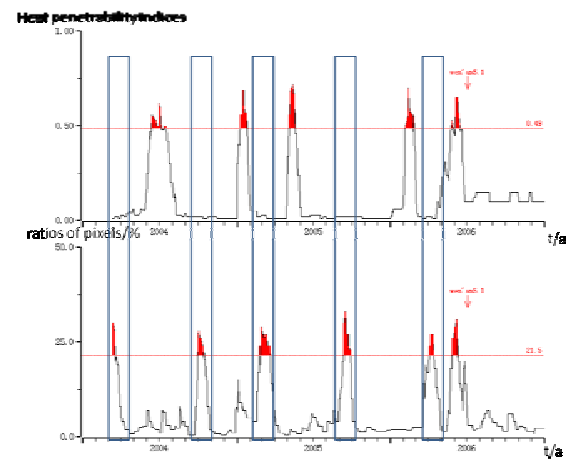

Fig.2. Heat penetrability indices and ratios of pixels with abnormal brightness temperature increasing

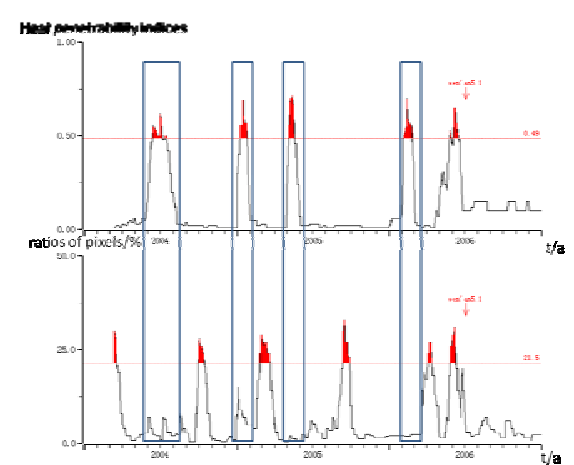

Fig.3. Heat penetrability indices and ratios of pixels with abnormal brightness temperature increasing 


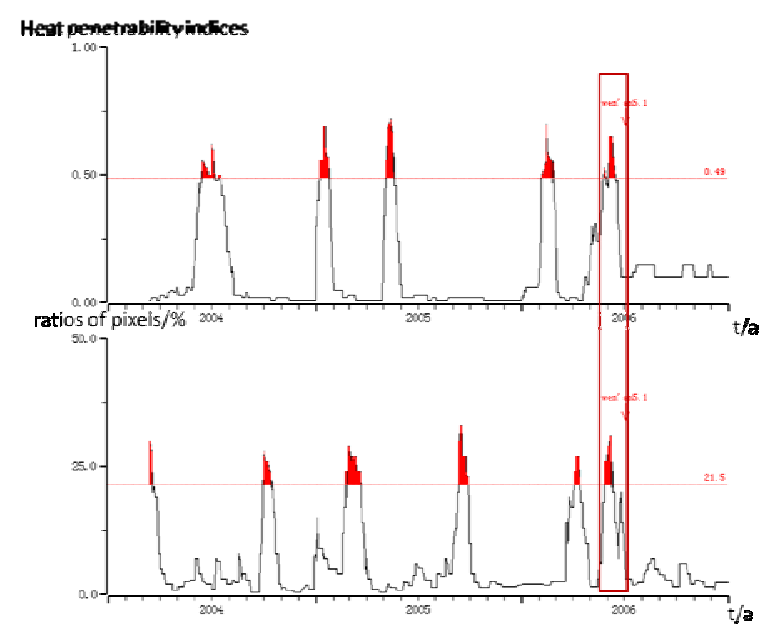

Fig.4. Heat penetrability indices and ratios of pixels with abnormal brightness temperature increasing

\section{Spatial Characteristics of ANOMAly}

When both the heat penetrability index and the anomaly ratio showed abnormal, it can be assumed a possible earthquake foreshadow. By analyzing the special distribution of the brightness temperature anomaly from the geostationary satellite infrared information, we can speculate on the spots where the earthquake possibly occurred.

Fig. 5 shows the distribution of the infrared brightness temperature change, one month before the Wen'an earthquake on June 15 th to July 15 th, 2006 . We can also get a basically clear sight of the rising and falling processes of the brightness temperature.

Pre-earthquake thermal anomalies, which could be obviously distributed along the faults, were mainly located in the epicenter and its west region. On 15 June 2006, 19 days before the Wen'an earthquake, there were a large range of brightness temperature anomalies of high value, which were most obviously seen in southwest of the epicenter. On 20 June, the thermal infrared anomalies drew gradually close to near the epicenter along the northeast direction, the range of which was attenuated and concentrated. On June 30th, only 4 days before the earthquake, the thermal infrared anomalies had already been very close up to the epicenter. On July 4th at noon, an MS5.1 earthquake occurred in Wen'an, Hebei. From July 5th to July 15 th, as we can see from Fig.5, the thermal anomalies gradually concentrated to the epicenter, among which the large-scale ones and those far from the epicenter slowly decreased and then disappeared. During the earthquake, the process of the thermal infrared anomaly change had experienced from large-range to concentrated then gradually to dissipation, and the maximum value appeared closely near the epicenter, which demonstrated well as the pre-earthquake anomaly.

\section{CONCLUSIONS}

MODIS thermal infrared data and geostationary satellite thermal infrared brightness temperature data were processed and analyzed of Wen'an earthquake. The results show that anomaly does exist before the earthquake in both the heat penetrability index and the anomaly ratio. With integrated analysis of these data, the earthquake prediction could be better. If those two showed abnormal, a moderate earthquake might happen in one month. Furthermore, if the anomaly ratio continued abnormal for a period of time, while the heat penetrability index unchanged, it's indicated that anomalies were often caused by temperature change. Therefore, this kind of anomalies shouldn't been considered as the pre-earthquake anomaly. Anomalies in the heat penetrability index explain the increase of tectonic activities, which cannot be equated with earthquakes, and then shouldn't been necessarily considered as the pre-earthquake anomaly either. The spatial change of the pre-earthquake anomaly mostly experienced the process from large-range to more concentrated then gradually to dissipation, and the forthcoming earthquakes usually occurred in concentration areas of anomalies.

\section{REFERENCES}

[1] Lv Q Q, Ding J H,Cui J K,etal. SATELLITE THERMAL INFRARED ANOMALY BEFORE THE ZHANGBEI EARTHQUAKE MS=6.2[J]. Earthquake. 1998,18(3):240-244.

[2] Ma Jin,Shan X J.AN ATTEMPT TO STUDY FAULT ACTIVITY USING REMOTE SENSING TECHNOLOGY - A CASE OF THE MANI EARTHQUAKE[J]. SEISMOLOGY AND GEOLOGY.2000,22(3):210-218.

[3] Kang C,Chen Z,Chen L,etal.Analysi on the satellite infrared anomaly feature before west to kunlun mountain pass M8.1 earthquake[J]. Northwestern Seismological Journal. 2003,25(1):12-15

[4] Guo X,Zhang Y S,Zhong M J.Study on Thermal Infrared Data of Satellite Remote Sensing Applied to Earthquake Prediction[J]. Northwestern Seismological Journal. 2005,27(3):223-227.

[5] Zhong M J,Zhang Y S.Thermal Infrared Anomaly before Pakistan Earthquake (M_S=7.8) on October 8,2005[J]. Northwestern Seismological Journal.2007,29(2):137-140.

[6] Zhang Y S,Guo X ,Zhang X M,etal.STUDY ON THE INVERSION METHOD OF LAND SURFACE TEMPERATURE BY APPLYING IR BRIGHT TEMPERATURE DATA OF STILL SATELLITE[J] Northwestern Seismological Journal.2004,26(2):113-117.

[7] Liu P X,Liu L Q,Chen S Y,etal.HEAT PENETRABILITY INDEX (HPI) METHOD FOR DISTILLING SUBSURFACE THERMAL INFORMATION FROM SATELLITE INFRARED IMAGES[J] Seismology and Geology. 2004,26(3):520-527.

[8] Zhang Y S, Guo X, Zhong M J, et al. Wenchuan earthquake: Brightness temperature changes from satellite infrared information. Chinese Science Bulletin, 2010,55(10):904-910

[9] NI Xiaoyin, LIN Shimin, CHEN Ying, etal. Analysis of Anomalies of MODIS Satellite Thermal Infrared Data in Fujian[J]. South China Journal of Seismology. 2008,28(4):93-103

[10] zhang Li-fang, Xu jie ,peng yan-ju, et al,2014,a study on neotectonic movement in the east china sea ,seismology and geology,36(3):692-705 

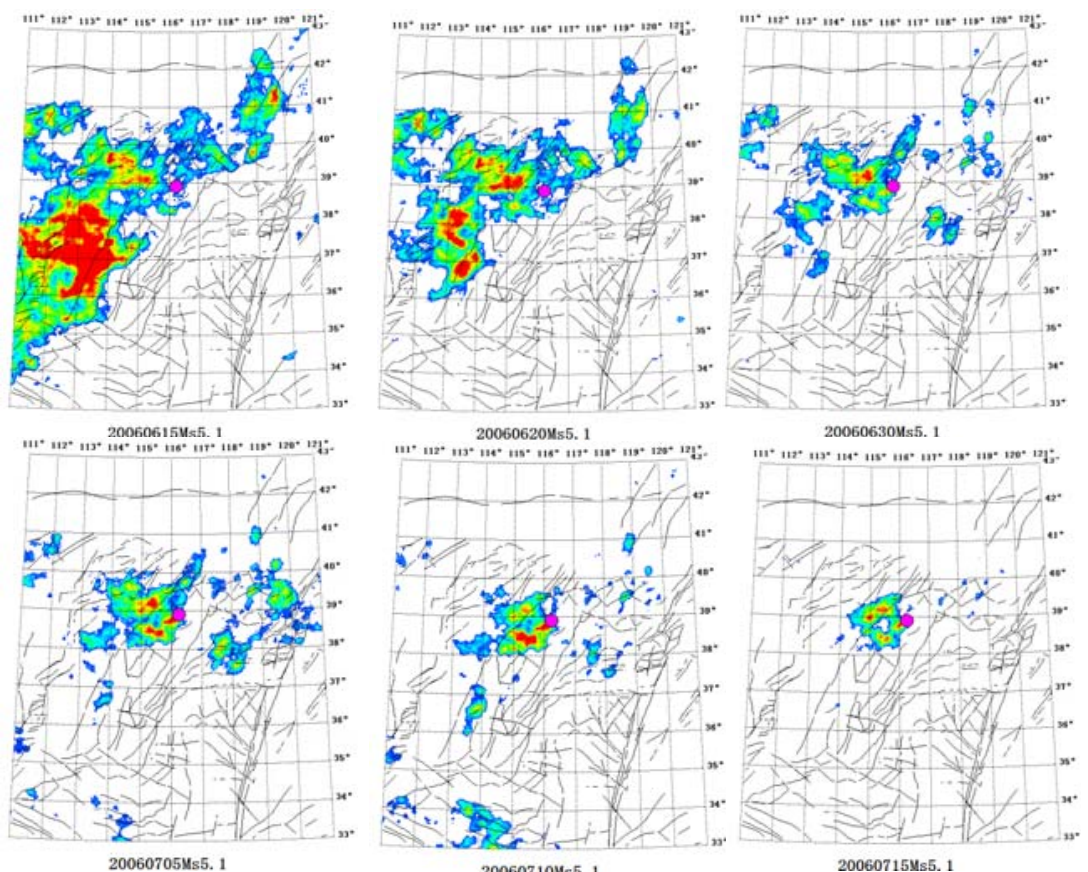

Fig.5. Distribution of thermal infrared anomalies in 15 June to 15 July2006 of Wen ' an 